\title{
FACTORS AFFECTING BREASTFEEDING PRACTICES APPLYING A CONCEPTUAL FRAMEWORK
}

\author{
Debra Hector*, Lesley King ${ }^{* *}$, Karen Webb ${ }^{*}$ \\ NSW Centre for Public Health Nutrition* \\ NSW Centre for Overweight and Obesity \\ University of Sydney
}

\section{Peter Heywood \\ World Bank}

A previous paper in this issue has described the considerable potential health benefits of breastfeeding. Despite this, survey data show that the majority of NSW women stop breastfeeding in the early months after birth, and most do not breastfeed exclusively for the recommended six months (see Allen and Hector, 'Benefits of breastfeeding', and Hector and Webb, 'Breastfeeding practices in NSW' in this issue). The planning of public health interventions to promote longer and more exclusive breastfeeding practices requires an understanding of the factors that affect breastfeeding (variously referred to as predictors, determinants, barriers, influences, and contributing factors). ${ }^{1}$

Our understanding of these factors, their relative importance, how they inter-relate, and how they respond to interventions is hampered by the lack of a coherent approach to research in this area. While there is a large body of published material on the factors affecting breastfeeding, most studies have focused on an extremely narrow range of factors, particularly the socio-demographic characteristics of mothers, and self-reported personal factors. This may be partly attributable to the ease of collecting these data, or the reliance on secondary analyses of 'factors' in data that have been collected for other purposes. A fundamental limitation of research to date is the lack of a conceptual or theoretical base; that is, there is no accepted, comprehensive overview of putative factors to guide researchers in planning their studies, or to assist readers to interpret results of studies in a broad context.

In this paper, we describe some of the limitations of the research on factors affecting breastfeeding. We also describe earlier attempts by ourselves and others to move towards developing a systematic approach to this area and propose a conceptual framework of factors affecting breastfeeding. The framework is intended for use in planning and organising future research and in designing and evaluating interventions to promote recommended breastfeeding practices.

\section{SOME LIMITATIONS OF RESEARCH}

Much published research has focused on the attributes of the mother and is often based on surveys that ask women why they didn't breastfeed or why they gave up breastfeeding early; that is, they ask women to provide a self-report of reasons. While the responses to these surveys are useful in identifying how women explain their infant feeding deci- sions, they are only the tip of the iceberg in terms of the underlying reasons why women don't follow recommended feeding practices. Surveys of why women stop breastfeeding provide only indirect and limited insight into the role of wider influences and interactions. Why is this?

First, women are unlikely to be aware of the many influences on their infant feeding behaviour, particularly the broader environmental and socio-cultural influences, such as lack of support. Second, women are often unable to articulate in survey responses, and/or are uncomfortable reporting, less socially acceptable ${ }^{2}$ or 'mother-driven' ${ }^{3}$ reasons (such as fear of loss of breast shape) for not breastfeeding or stopping breastfeeding early; they tend to report more child-centred reasons ${ }^{3}$ such as 'child did not want the breast' or reasons beyond the mother's control, notably 'insufficient milk ${ }^{4-9}$. 'Insufficient milk' is one of the commonest reasons women give for stopping breastfeeding, yet evidence indicates that less than 5 per cent of women are physiologically incapable of producing an adequate supply of milk..$^{7,10,11}$ Insufficient milk usually results from the woman not breastfeeding frequently enough or long enough, which, in turn, may be affected by circumstances such as the mother returning to work (and working in an environment which does not facilitate expressing breastmilk), or being busy with other siblings, family disruption or dysfunction, or a young mother's desire to minimise time spent in care giving. The explanation of 'insufficient milk' therefore masks a range of underlying factors that undermine breastfeeding.

Survey findings tend to highlight one particular factor or several factors as being particularly important. However, if there are sufficient factors encouraging breastfeeding, any one barrier to breastfeeding may not preclude breastfeeding. ${ }^{7}$ For example, whilst painful nipples are a relatively common reason given for stopping breastfeeding, the majority of women with painful nipples continue breastfeeding. ${ }^{9}$ A critical chain of events may lead to a woman stopping breastfeeding early. ${ }^{7,12,13}$

\section{TOWARDS A SYSTEMATIC APPROACH}

The lack of a systematic approach to selecting factors for investigation in 'determinants' research in breastfeeding has been observed by several researchers. ${ }^{14-16}$ As noted by Scott et al, "no two studies investigate the same factors using comparable methods, thus making it impossible to identify common factors across studies that may be worthy of more inquiry'. ${ }^{14}$ The majority of studies implicitly focus on a particular subset of factors, usually those socio-demographic attributes of the mother and family, without due acknowledgement of the wide range of additional potential influences. The socio-demographic characteristics of the mother are actually 'risk markers', those factors that 
signal where a problem is occurring, but may not directly contribute to it. ${ }^{17}$

A systematic approach to research is therefore required to gain a complete picture of the issues surrounding improved breastfeeding practices, and to identify factors that may be modifiable for intervention planning. A number of researchers have identified lists of possible factors affecting breastfeeding practices, and/or have grouped or categorised these factors. ${ }^{16,18,19}$ In our earlier reports ${ }^{1,20}$, we suggested a set of categories for classifying factors, adapted from extensive research on influences on patient compliance with health care regimens (see Table 1). ${ }^{21,22}$

Since then, we have considered a variety of theories that have helped to refine our thinking about the range of factors which influence breastfeeding. Tiedje and others, in their study of influences on mothers feeding decisions, proposed an adaptation of an 'ecological model', in which influences are seen as emanating from relationships between people and groups, and their environments. ${ }^{23}$ We extended the focus on environments and the wider social contexts of

\section{TABLE 1}

\section{CATEGORIES OF FACTORS AFFECTING BREASTFEEDING PRACTICES}

Socio-demographic characteristics of the mother and family

Structural and social support

Health and risk status of mothers and infants

Mothers' knowledge, attitudes and skills

Aspects of the feeding regime/practices

Health services (including hospital and health facili-

ties) organisation, policies and practices

Socio-cultural, economic and environmental factors

Adapted from Sackett and Haynes ${ }^{21,22}$

behaviour by considering planning frameworks for health promotion interventions, in particular the Ottawa Charter for Health Promotion. ${ }^{24}$ This identifies five types of interventions: developing personal skills, reorienting health services, creating supportive environments, developing

FIGURE 1

A CONCEPTUAL FRAMEWORK OF FACTORS AFFECTING BREASTFEEDING PRACTICES

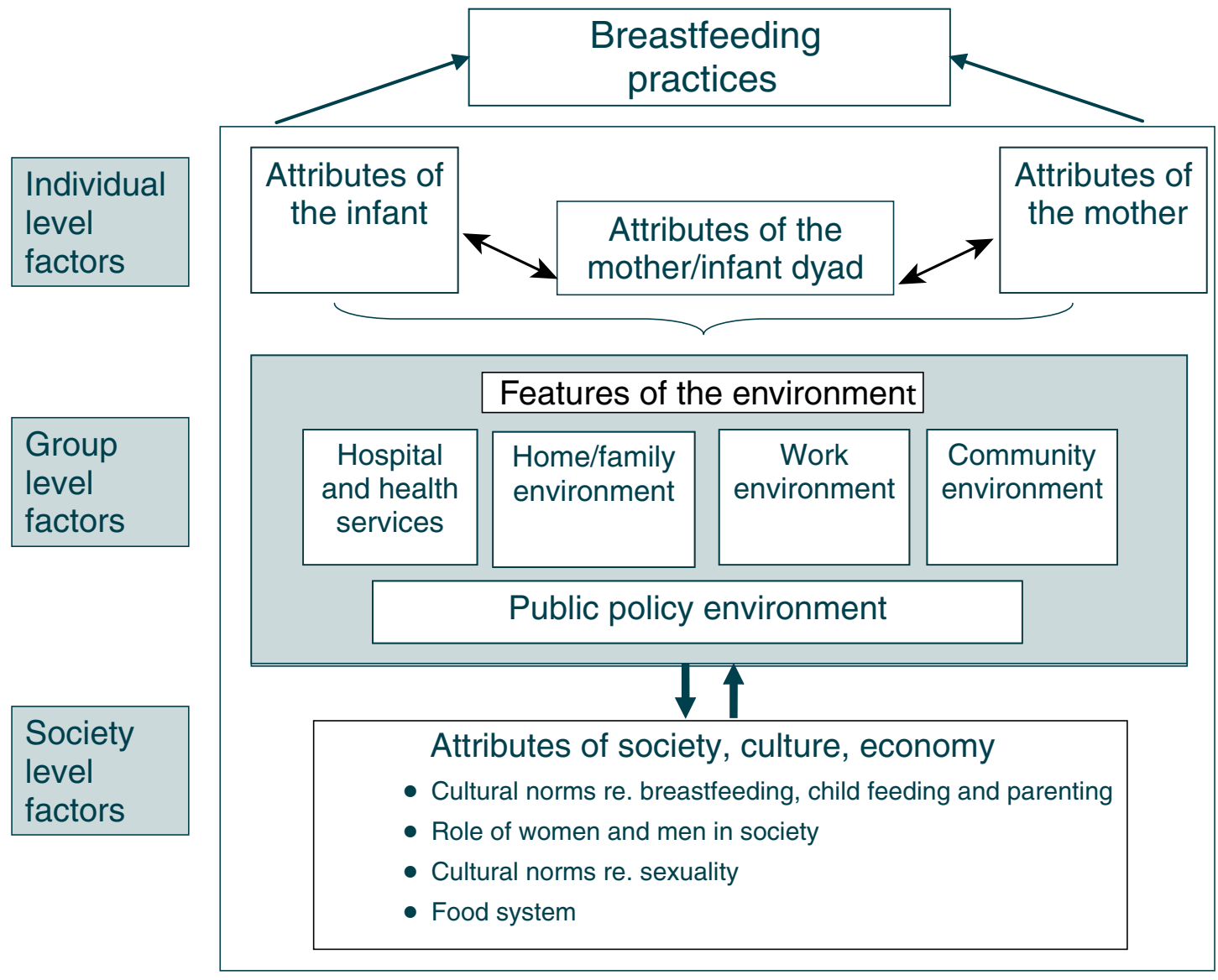


healthy public policy and strengthening community action. Such actions are variously directed to modifying individual and personal factors, but more so to modify the environments in which individuals live and breastfeed.

Consequently, we developed a conceptual framework for understanding the influences on breastfeeding that incorporates a variety of elements of relevant theories for understanding health behaviour and for planning effective public health interventions to influence health behaviour.

\section{CONCEPTUAL FRAMEWORK OF FACTORS AFFECTING BREASTFEEDING}

The conceptual framework (Figure 1) proposes three levels of factors that influence breastfeeding practices: individual, group and society. The framework can be used to generate hypotheses about factors affecting breastfeeding and the types of interventions that might be used to address them.

Individual level factors relate directly to the mother, infant, and the 'mother-infant dyad'. They include the mother's intention to breastfeed, her knowledge, skills and parenting experience, the birth experience, health and risk status of mothers and infants, and the nature of early interaction between mother and infant. Each of these can directly influence the initiation and duration of breastfeeding, and are frequently correlated with social and demographic variables.

Group level factors are the attributes of the environments in which mothers and infants find themselves, the attributes that enable mothers to breastfeed. Environments with a direct influence on mothers and infants include:

- the hospital and health facilities environment, in which practices and procedures such as infants routinely rooming-in with mothers to allow demand feeding, postpartum skin-to-skin contact and providing professional support with breastfeeding technique difficulties influence the early feeding experience and the follow-up care and support,

- the home and peer environment, where physical and social factors such as size of household, parity, family circumstances, partner attitudes and support, and peer support affect the time, energy and resolve that mothers have for breastfeeding

- the work environment, in which policies, practices and facilities such as work hours and flexibility, facilities and policies that enable on-site expressing and storing of breastmilk influence mother's ability to combine work and breastfeeding

- the community environment, which signals the extent to which breastfeeding is recognised as a norm, and reinforced by facilities and policies in public places, for example parenting rooms in shopping centres and entertainment venues, 'breastfeeding friendly' public transport, restaurants
- the public policy environment, which modifies how each of these environments influence mother's feeding decisions. For example, benefits such as maternity and paternity leave, childcare allowances and health insurance have a significant impact on the hospital, home, and work environments that in turn, influence infant feeding decisions directly.

Societal level factors influence the acceptability and expectations about breastfeeding and provide the background or the context in which mothers' feeding practices occur. These include cultural norms regarding breastfeeding, child feeding, and parenting; the role of women in society, including how working outside the home is valued; the extent to which men's social role includes support for breastfeeding mothers; the extent to which exposing breasts for feeding is complicated by cultural norms regarding sexuality; and the economic importance of products such as breastmilk substitutes and complementary foods in the food system.

Group level and societal level influences may interact in either positive or negative ways with maternal knowledge and skills. For example, a mother may be predisposed to breastfeed, but a non-supportive environment in the hospital may lead to her deciding to stop breastfeeding early. Similarly, even if breastfeeding is still occurring at hospitaldischarge, a lack of support at home or in the community may also lead to her stopping early. Again, broader societal attitudes about sexuality, and especially breasts, can influence the manner and degree of community support.

\section{IMPLICATIONS FOR INTERVENTIONS RESEARCH}

Factors operating at various levels may negate one another, so a broader view is required when designing attempts to improve breastfeeding rates and duration. Thus, interventions directed only at persuading mothers to breastfeed may fail if they are not complemented by other interventions that support her in hospital, at home, and at work. Indeed, aiming interventions at any one factor affecting breastfeeding is likely to be less successful in achieving and maintaining change than those addressing multiple factors, across several categories, and at several levels.

The main purpose of research on factors affecting breastfeeding is to inform the design of interventions. As well, the results of intervention research can provide insights about significant influences on breastfeeding. Factors that cannot be elucidated by qualitative or cross-sectional studies, such as the effect of health professional training, or changes to health service practices (for example the Baby Friendly Hospital Initiative), can be determined to some extent by the success, or otherwise, of methodologically sound, well-evaluated interventions.

\section{SUMMARY}

Knowledge about factors affecting breastfeeding can be developed by further research on underlying factors and 
by drawing out the implications and lessons from intervention research. The use of a conceptual framework to guide this research and the interpretation of results can help us to understand the relative importance of different factors, and how they interact, in turn, helping us to design effective interventions.

\section{REFERENCES}

1. Hector D, King L, Webb K. State of food and nutrition in NSW series: Overview of recent reviews of interventions to promote and support breastfeeding. Sydney: NSW Department of Health; 2004.

2. Rempel L. Breastfeeding reasons predict breastfeeding decisions. University of Queensland Family Centre Newsletter March 2000.

3. McLennan JD. Early termination of breast-feeding in periurban Santo Domingo, Dominican Republic: mothers' community perceptions and personal practices. Rev Panam Salud Publica 2001; 9(6): 362-367.

4. Dykes F, Williams C. Falling by the wayside: a phenomenological exploration of perceived breast-milk inadequacy in lactating women. Midwifery 1999; 15(4): 232-246.

5. Arora S, McJunkin C, Wehrer J, Huhn P. Major factors influencing breastfeeding rates: Mother's perception of father's attitude and milk supply. Pediatrics 2000; 106(5): E67.

6. McCarter-Spaulding DE, Kearney MH. Parenting self-efficacy and perception of insufficient breast milk. JOGN Nurs 2001; 30(5): 515-522.

7. Binns CW, Scott JA. Breastfeeding: reasons for starting, reasons for stopping and problems along the way. Breastfeeding Rev 2002; 10(2): 13-19.

8. Blyth R, Creedy DK, Dennis CL, Moyle W, Pratt J, De Vries SM. Effect of maternal confidence on breastfeeding duration: an application of breastfeeding self-efficacy theory. Birth 2002; 29(4): 278-284.

9. Cooke M, Sheehan A, Schmied V. A description of the relationship between breastfeeding experiences, breastfeeding satisfaction, and weaning in the first 3 months after birth. J Hum Lact 2003; 19(2): 145-156.
10. McVeagh P. Breastfeeding - help! Med Today April 2001; 20-29.

11. Renfrew MJ, Woolridge MW, McGill HR. Enabling women to breastfeed. London. The Stationery Office; 2000.

12. Sjolin S, Hofvander Y, Hillervik C. Factors related to early termination of breastfeeding. A retrospective study in Sweden. Acta Paediatr Scand 1977; 66(4): 505-511.

13. Hoddinott P, Pill R. Qualitative study of decisions about infant feeding among women in east end of London. BMJ 1999; 318: 30-34.

14. Scott JA, Binns C. Factors associated with the initiation and duration of breastfeeding: a review of the literature. Aust $J$ Nutr Diet 1998; 55(2): 51-61.

15. Scott JA, Landers MC, Hughes RM, Binns CW. Factors associated with breastfeeding at discharge and duration of breastfeeding. J Paediatr Child Health 2001; 37: 254-261.

16. Kirkland VL, Fein SB. Characterizing reasons for breastfeeding cessation throughout the first year postpartum using the construct of thriving. J Hum Lact 2003; 19: 278-285.

17. Hawe P, Degeling D, Hall J. Evaluating health promotion: a worker's guide. Maclennan and Petty, Sydney; 1990.

18. Arbon S, Byrne J. The reliability of a breastfeeding questionnaire. Breastfeeding Rev 2001; 9(2): 23-31.

19. Dennis CL. Breastfeeding initiation and duration: a 19902000 literature review. JOGN Nurs 2002; 31: 12-32.

20. Hector D, Webb K, Lymer S. State of food and nutrition in NSW series: Report on breastfeeding in NSW. Sydney. NSW Department of Health: 2004 (Revised 2005).

21. Sackett DL, Haynes RB. Compliance with therapeutic regimens. Baltimore MD: Johns Hopkins University Press; 1976.

22. Haynes RB, Taylor DL, Sackett DL (Eds.). Compliance in health care. Baltimore, MD: Johns Hopkins University Press; 1979.

23. Tiedje LB, Schiffman R, Omar M, Wright J, Buzzitta C, McCann A et al. An ecological approach to breastfeeding. MCN, The American Journal of Maternal Child Nursing 2002; 27 (3):154-60.

24. Ottawa Charter for Health Promotion 1986 1st International Conference on Health Promotion, Ottawa, Canada, 21 November 1986-WHO/HPR/HEP/95.1 1 궁. 